\title{
A prehabilitation program included in an ERAS protocol for radical cystectomy
}

\author{
$\underline{\text { C. Marín Zaldívar }}{ }^{1}$, R. Casans FrancéS², J. Subirá RíOs², J. GuilléN AntóN², J. LongáS Valien², J. M. Calvo \\ Vecino \\ ${ }^{11}$ Hospital Universitario Salamanca - Salamanca (Spain), ${ }^{2}$ Hospital Clínico Universitario Lozano Blesa - \\ Zaragoza (Spain)
}

\section{Background and Goal of Study:}

Bladder cancer and its surgical treatment by radical cystectomy have a high morbidity and mortality. The goal of this study is to know if our prehabilitation program decreases the postoperative complications and hospital stay by optimizing the physical and mental conditions before the surgery.

\section{Materials and Methods:}

A prospective cohort of patients included in the prehabilitation program (PREHAB, january 2015 - march 2017) was compared to an historical cohort receiving no prehabilitation (NO-PREHAB, january 2012 - december 2014), both of them following enhancing recovering protocols.

The program is based on physical therapy with aerobic and resistance exercises, respiratory exercises, nutritional supplementation according to the patient's condition and malnutrition risk (MUST toolkit) as well as cognitive therapy.

The discrete variables were described as frequency (percentage) and the continuous variables as a median (interquartile range), with Fisher's test (discrete) and Wilcoxon's (continuous). A p value $<0.05$ was considered statistically significant. Multivariate analysis was made for age, Charlson Comorbidity Index, prehabilitation, surgical approach, haemoglobin and bleeding during the surgery versus total complications and major complications (Clavien-Dindo $>2, C D>2$ ) at 30 days after surgery.

\section{Results and Discussion:}

The sample included 141 patients, 101 in the historical cohort (NO-PREHAB) and 40 in the prospective cohort (PREHAB). Significant differences were found between the two groups according to the surgical approach, with more laparoscopy approach in the PREHAB group (66.66\% vs $14,89 \%$ NO-PREHAB; $p<0.0001$ ).

There was no difference in mortality and re-entry rate.

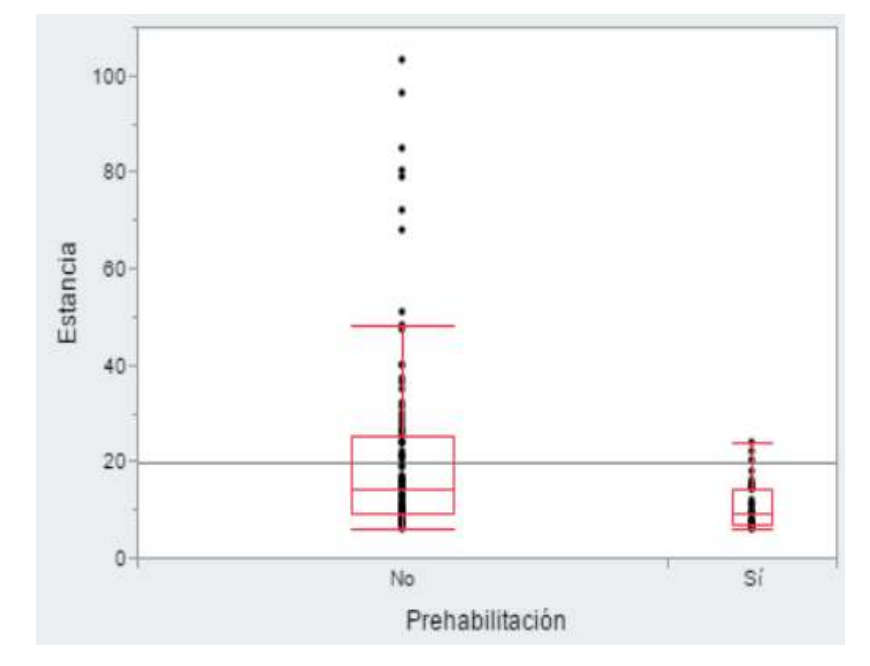

Hospital stay:

PREHAB 8 (9.2-11.5) vs NOPREHAB $14(9-25.25)(p=0.0006)$

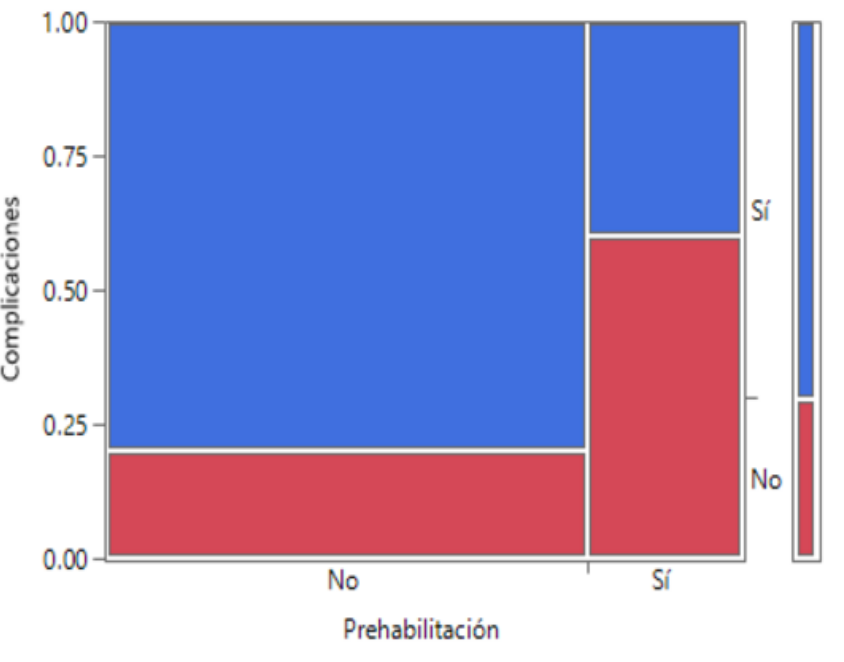

Total complications within 30 days: PREHAB 12 (40\%) vs NO-PREHAB $75(79,79 \%)(p<0.0001)$

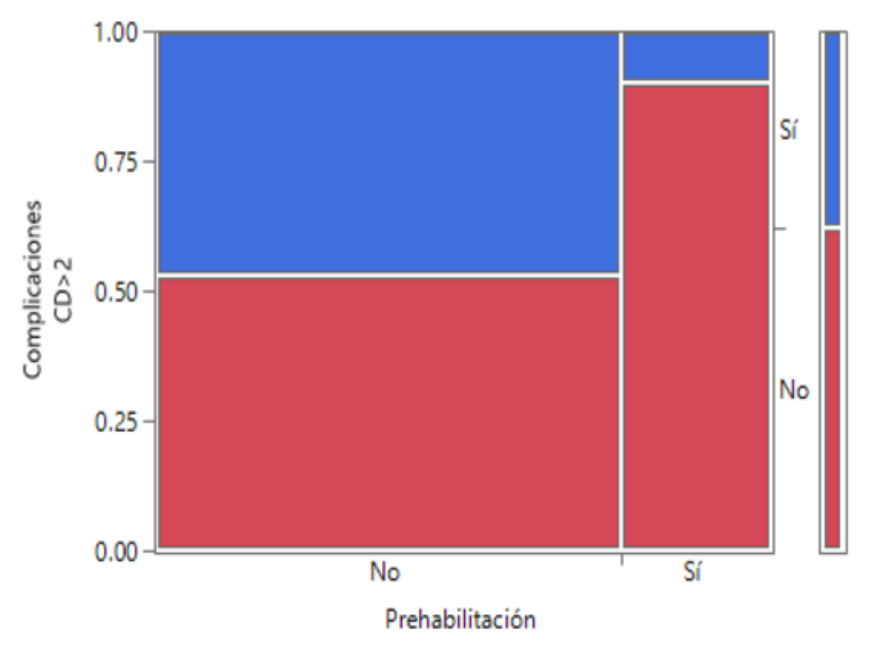

Major complications $C D>2$ : PREHAB 3 (10\%) vs NOPREHAB $44(46,8 \%)(p<0.0001)$

The multivariate analysis did not show correlation between major complications and laparoscopy [-0.144($0.313,0.032) ; \mathbf{p}=\mathbf{0 . 1 8 8}]$; however it showed correlation with prehabilitation $[-\mathbf{0 . 3 2 4}(-0.474,-0.157) ; p=0.0026]$

\section{Conclusion(s):}

This Prehabilitation Program improved the outcomes of patients undergoing radical cystectomy by reducing hospital stay and postoperative complications, specially major complications. More studies are needed to determine the influence of prehabilitation and laparoscopy on the outcomes of radical cystectomy. 